\title{
Synthesis of Dendrimer Assisted Co-Pd Bimetallic Nanoparticles and Catalytic Activity for Suzuki-Miyaura Coupling Reactions
}

\author{
Mohammad Sayedul Islam*, Mohammad Wahab Khan \\ Email address: \\ sayedulbuet98@gmail.com (M. S. Islam),mwkhan@chem.buet.ac.bd (M. W. Khan) \\ ${ }^{*}$ Corresponding author
}

Department of Chemistry, Faculty of Engineering, Bangladesh University of Engineering and Technology (BUET), Dhaka, Bangladesh

To cite this article:

Mohammad Sayedul Islam, Mohammad Wahab Khan. Synthesis of Dendrimer Assisted Co-Pd Bimetallic Nanoparticles and Catalytic Activity for Suzuki-Miyaura Coupling Reactions. American Journal of Nanosciences. Vol. 5, No. 1, 2019, pp. 1-8.

doi: $10.11648 /$ j.ajn.20190501.11

Received: May 31, 2019; Accepted: July 3, 2019; Published: July 22, 2019

\begin{abstract}
Triazine-based dendrimer 2,4,6-tris(di-acetamido)-1,3,5-triazine encapsulated heterogeneous Co-Pd bimetallic nanoparticles (NPS) were prepared through sequential loading method from the dendrimer which was synthesized directly between the reaction of 2,4,6-triamino-1,3,5-triazine and acetyl chloride at room temperature about 2 hours. The physicochemical characteristics of Co-Pd bimetallic NPS were studied with the aid of EDX, XRD, TG\& DSC techniques. The presence of cobalt was $55.75 \%$ and palladium was $9.40 \%$ of the mass of the 2,4,6-tris(di-acetamido)-1,3,5-triazine assisted CoPd Bimetallic NPs 4 observed from EDX analysis. The TG\& DSC analysis curves indicated the thermal stability up to $320^{\circ} \mathrm{C}$ which is a very important steadiness key for catalytic effectiveness of the prepared Co-Pd Bimetallic NPs 4. The nano-shape FCC structure of Co-Pd bimetallic NPs was confirmed by XRD analysis. The average size of Co-Pd bimetallic NPs of 17.50 $\mathrm{nm}$ length was calculated through the Debye-Scherrer formula while TEM analysis showed the average particle size was 14.50 $\mathrm{nm}$. The dendrimer was also analyzed by IR, ${ }^{1} \mathrm{HNMR},{ }^{13} \mathrm{CNMR}$ and elemental analysis. The catalytic performance of these nanoparticles' catalyst was explored for $\mathrm{C}-\mathrm{C}$ coupling reactions such as Suzuki-Miyaura at moderate reaction conditions whereas the reusing potential of the catalyst was evaluated without noticeable loss of activity for five successive runs. All the results of the analysis of this catalyst make it a cost-effective and highly active catalyst for the carbon-carbon cross-coupling reactions which are demand in chemical and pharmaceutical industries.
\end{abstract}

Keywords: Bimetallic Nanoparticles (NPs), Triazine, Dendrimer and Suzuki-Miyaura Reaction

\section{Introduction}

Catalytic $\mathrm{C}-\mathrm{C}$ bond forming reactions provide all reactive synthetic routes to a variety of important chemicals and pharmaceuticals of their building blocks. The advent of nanotechnology for the production of metal nanoparticles opens up new possibilities for a possible revolution in the field of catalysis. Dendrimers are highly branched macromolecules and are generally described as a spherical structure with a high degree of symmetry [1-2]. Dendrimers are also considered to have new types of hosts for containing guest molecules due to their three-dimensional structure with the internal cavity receiving space, so many metal nanoparticles have been successfully synthesized using dendrimer as a template [3-5]. The driving force for encapsulation within host dendrimer may be based on electrostatic interactions, complexation reactions, steric confinement, various types of weak forces (van der Waals, hydrogen bonding, hydrophobic force, etc.) and their combinations [6-7].

As a result the preparation technique for monometallic or bimetallic nanoparticles via dendrimer templates [8-9] has at least five potential benefits: (i) with relatively uniform composition and structure; dendrimer patterns themselves afford distinct nanoparticle replicas; (ii) nanoparticles area unit stabilized by encapsulation in dendrimers and thus don't agglomerate throughout catalytic reactions; (iii) the nanoparticles are mainly retained in the dendrimers by steric effects, and therefore, a substantial portion of their surface is 
not passivated and available for participation in catalytic reactions; (iv) the dendrimer branches can be used as selective gates to control the access of molecules (substrates) to the encapsulated (catalytic) nanoparticles; and (v) the dendrimer periphery can be tailored to control the solubility of the hybrid nanocomposite and to be used as a handle to facilitate linkage with surfaces and other polymers. However, bimetallic dendrimer-encapsulated nanoparticles have been receiving a significant amount of attention due to their promising properties, unique characteristics and novel applications in catalysis and other advanced nano-science and technology areas [10]. Bimetallic nanoparticles based on $\mathrm{Pt}, \mathrm{Pd}$, or $\mathrm{Rh}$ is that $3 \mathrm{~d}$ transition metals are abounding, and low priced, and can be acclimated to alter big-ticket blue-blooded metals in catalytic processes [11-12]. Nano cobalt supported nanopalladium catalyst obtained by an innovative methodology of nanoparticle transfer from the intermediate carrier metallodendrimer to the target metallic element carrier was an extremely economical and selective catalyst, giving the maximum amount as quantitative conversion and yield during a series of Suzuki-Miyaura reaction, Sonogashira reaction [13]. Indeed, catalysts for bimetallic nanoparticles incorporated in/on insoluble endorses with ligand-free and marginal or no leaching are essential from the point of view of both reducing environmental burdens and value-efficiency. Constitutional simplicity, standardized diffusion, reduced cost and dependable dendrimeric surface, improved efficiency and life cycle remain the significant challenges for a heterogeneous catalyst.

Moreover, to the best of our ability, no research can be acquired in the literature on the synthesis of melamine-based dendrimer embedded bimetallic Co-Pd NPs including the use of their heterogeneous catalytic activity in Suzuki-Miyaura cross-coupling reactions. Here, we have reported the synthesis, characterization, and utilization as a catalyst bimetallic Co-Pd NPs.

\section{Experimental}

\subsection{Materials and Methods}

All reagents such as 2,4,6-triamino-1,3,5-triazine, acetyl chloride, phenylboronic acid, $\mathrm{CoCl}_{2} \cdot 6 \mathrm{H}_{2} \mathrm{O}$, and $\mathrm{PdCl}_{2}$ as well as all solvents such as DMF, DMSO, $\mathrm{CH}_{3} \mathrm{CN}, \mathrm{C}_{2} \mathrm{H}_{5} \mathrm{OH}$, DMF, and bases such as $\mathrm{NaOH}, \mathrm{KOH}, \mathrm{KO}^{t} \mathrm{Bu}, \mathrm{Na}_{2} \mathrm{CO}_{3}$, and $\mathrm{Et}_{3} \mathrm{~N}$, were purchased from Aldrich and used directly. The IR spectrum was taken with $\mathrm{KBr}$ pellets on a Shimadzu FTIR 8400S Fourier Transform. On the BRUKER DPX-400 spectrophotometer, NMR spectra were taken using tetramethylsilane as an internal standard. The chromatography of the analytical thin layer (TLC) was silica gel, 60 F 254 coated on 25 sheets of aluminum TCC ( 20 / 20 $\mathrm{cm}$ ). On silica gel $60 \mathrm{~N}$ (neutral, 40-100 $\mu \mathrm{M}$ ) silica gel column chromatographic separations were made. The thermal stability of nanoparticles was ascertained by a thermogravimetric analyzer (NETZSCH STA 449F3) from 26 to $600^{\circ} \mathrm{C}$. TG and DSC data were detected in a nitrogen atmosphere using crisp aluminum oxide at $10^{\circ} \mathrm{k} / \mathrm{min}$ heating rates and 40 and $60 \mathrm{~mL} / \mathrm{min}$ flow rates. SEM and EDX of Co-Pd bimetallic NPs 4 were taken by the JEOLJSM-7600F. A PANANALYTICAL X-ray diffractometer was also used to define particle size and phase. TEM analysis was accomplished by Philips CM12 transmission electron microscope (operating at $200 \mathrm{KeV}$ ).

\subsection{Synthesis of Dendrimer 3 and Dendrimer 3 Assisted Co-Pd bimetallic Nanoparticles 4}

\subsubsection{Synthesis of 2,4,6-tris(di-acetamido)-1,3,5-triazine (Dendrimer 3)}

The mixture of $0.3 \mathrm{~g}(0.00237 \mathrm{~mol})$ 2,4,6-triamino-1,3,5triazine, $4 \mathrm{~mL}$ of acetyl chloride was added to $8 \mathrm{~mL}$ of DMSO and the reaction mixture was stirred in a $250 \mathrm{~mL}$ round bottom flask at room temperature for about 2 hours. The reaction was observed by TLC. The reaction was stopped by introducing distilled water after the perfect transformation of the reactants into products. The reaction combination with $\mathrm{CHCl}_{3}$ was then retrieved. After the solvent was removed, the product was crystallized using ethanol and the required compound 3 was found as white crystalline solid, m. p. $137-139^{\circ} \mathrm{C}$, White crystalline solid; yield: 95\%, IR (KBr): $\mathrm{v}_{\max } 3035,2965,1687,1675,1620$, 1530, 1360 and $1290 \mathrm{~cm}^{-1} .{ }^{1} \mathrm{H}$ NMR (400 MHz, $\left.\mathrm{D}_{2} \mathrm{O}\right): 2.40$ (s, 18H) ppm. ${ }^{13} \mathrm{C}$ NMR (100 MHz, $\left.\mathrm{D}_{2} \mathrm{O}\right): 66.83,88.34$, 97.34, 151.83 and 161.44 ppm. Anal. Calcd. for $\mathrm{C}_{16} \mathrm{H}_{19} \mathrm{~N}_{5} \mathrm{O}_{6}$; C 47.62, H 4.80, N 22.21; found: C 47.58, H 4.78, N 22.15

\subsubsection{Synthesis of 2,4,6-tris(di-acetamido)-1,3,5-triazine, 3 Assisted Co-Pd Bimetallic NPs 4}

In $\mathrm{CH}_{3} \mathrm{CN}$ (12 mL), $0.200 \mathrm{~g} \mathrm{2,4,6-tris(di-acetamido)-1,3,5-}$ triazine 3 (0.00054 mmol), $0.00096 \mathrm{~g} \mathrm{PdCl}_{2}, \mathrm{NH}_{2}-\mathrm{NH}_{2} \cdot \mathrm{H}_{2} \mathrm{O}(1.5$ mmol) were stirred for 1 hour under reflux in a round bottom flask at $80^{\circ} \mathrm{C}$. Then $0.01536 \mathrm{~g}$ of $\mathrm{CoCl}_{2} \cdot 2 \mathrm{H}_{2} \mathrm{O}$ and $\mathrm{NH}_{2}-$ $\mathrm{NH}_{2} \cdot \mathrm{H}_{2} \mathrm{O}$ were added to the reaction mixture dropwise to a certain extent exceeding the equivalent amount of copper salt. In order to maintain $\mathrm{pH} 11$, the $\mathrm{NaOH}$ solution $(0.3 \mathrm{M})$ was gradually added to the reaction mixture and the suspension was stirred with a magnetic stirring bar at $80^{\circ} \mathrm{C}$ for 2 hours. After the reaction mixture had been settled, the black residue originated. The residue was filtered after the reaction mixture was centrifuged (4000 rpm for $20 \mathrm{~min}$ ), washed with distilled water and acetone and dried overnight at $120^{\circ} \mathrm{C}$ in an oven.

\subsection{Application of 2,4,6-tris(di-acetamido)-1,3,5-triazine, 3 Assisted Co-Pd Bimetallic NPs 4 in the Suzuki-Miyaura Coupling Reactions}

Co-Pd bimetallic NPs 4 (1.0 mol\%), aryl halides (1.0 $\mathrm{mmol})$, phenylboronic acid $(1.2 \mathrm{mmol}), \mathrm{Na}_{2} \mathrm{CO}_{3}(1 \mathrm{mmol})$, and $5 \mathrm{~mL}$ of $\mathrm{EtOH} / \mathrm{H}_{2} \mathrm{O}(1: 4 \mathrm{v} / \mathrm{v})$ were stirred about $1 \mathrm{~h}$ at $70^{\circ} \mathrm{C}$ to a round bottom flask. With the aid of TLC chromatography, the reaction was monitored and after completing the reaction, the solvent was removed through vacuum rotary evaporator. The residue was extracted with $\mathrm{CHCl}_{3}$ as well as purified by column chromatography using ethyl acetate/hexane $(1: 5 \mathrm{v} / \mathrm{v})$. 


\subsubsection{Synthesis of Biphenyl 11}

pale yellowish, yield \% (table 2), solid, mp. $66-68^{\circ} \mathrm{C}$ ( lit. $\left.69.2^{\circ} \mathrm{C}\right)[14]$

IR (KBr): $v_{\max } 3017.71, \quad 1597.65,1483.53,1435.52$, 1308.43, 1217.27, 977.13, 800.65

${ }^{1} \mathrm{H}$ NMR (400 MHz, $\left.\mathrm{CDCl}_{3}\right), \delta 7.63(\mathrm{dd}, J=7.2 \mathrm{~Hz}, 1.6 \mathrm{~Hz}$, 2H), 7.54 (t, $J=7.2 \mathrm{~Hz}, 4 \mathrm{H}$ ), 7.34 ( dd, $J=5.6 \mathrm{~Hz}, 1.2 \mathrm{~Hz}$, $4 \mathrm{H}),{ }^{13} \mathrm{C}$ NMR (100 MHz, $\left.\mathrm{CDCl}_{3}\right): \delta 141.30,128.55,127.78$, 127.26.

\subsubsection{Synthesis of 4-Methyl-biphenyl 12}

white crystalline solid, yield $\%$, (table 2 ), mp. $44-46^{\circ} \mathrm{C}$ (lit. $\left.44-47^{\circ} \mathrm{C}\right)[14]$

IR (KBr): $v_{\max }$ 2922.24, 1488.40, 1445.10, 1403.50, $1378.54,1216.65,823.43,755.67$

${ }^{1} \mathrm{H}$ NMR $\left(400 \mathrm{MHz}, \mathrm{CDCl}_{3}\right): \delta 2.28(\mathrm{~S}, 3 \mathrm{H}), 7.25(\mathrm{~d}, J=$ $8.4 \mathrm{~Hz}, 2 \mathrm{H}), 7.31-7.34(\mathrm{~m}, 1 \mathrm{H}), 7.35-7.44(\mathrm{~m}, 2 \mathrm{H}), 7.58$ (d, $J=8.0 \mathrm{~Hz}, 2 \mathrm{H}),{ }^{13} \mathrm{C} \mathrm{NMR}\left(100 \mathrm{MHz}, \mathrm{CDCl}_{3}\right): \delta$ 21.60, $127.25,127.95,128.10,129.20,137.60,138.00,141.90$

\subsubsection{Synthesis of 4-Methoxy-biphenyl 13}

white crystalline solid, yield $\%$, (table 2 ), mp. $83-85^{\circ} \mathrm{C}$ (lit. $\left.86-90^{\circ} \mathrm{C}\right)[14]$
IR (KBr): $v_{\max } 3020.65,1613.63,1605.65,1519.92$, $1487.52,1216.65,909.13,759.65$

${ }^{1} \mathrm{H}$ NMR $\left(400 \mathrm{MHz}, \mathrm{CDCl}_{3}\right): \delta 6.81(\mathrm{~d}, J=7.2 \mathrm{~Hz}, 3 \mathrm{H})$, 7.49 (t, $J=7.2 \mathrm{~Hz}, 1 \mathrm{H}), 7.54$ (t, $J=7.2 \mathrm{~Hz}, 2 \mathrm{H}), 7.57-5.70$ (m, $4 \mathrm{H}), 3.38(\mathrm{~S}, 3 \mathrm{H}) .{ }^{13} \mathrm{C} \mathrm{NMR}\left(100 \mathrm{MHz}, \mathrm{CDCl}_{3}\right): \delta 55.21$, $114.11,126.20,126.40,128.20,128.40,133.21,140.61$, 159.22

\section{Result and Discussions}

\subsection{Synthesis and Characterization}

Dendrimer 3 was produced by a reaction of $0.3 \mathrm{~g}(0.00237$ mol) 2,4,6-triamino-1,3,5-triazine 1 with $4 \mathrm{~mL}$ of acetyl chloride in anhydrous DMSO for 2 hours in a nitrogen atmosphere at room temperature (Figure 4). The growth of the reaction was monitored by thin layer chromatography (TLC) and the solid reaction mixture was washed with sodium bicarbonate solution after completion of the reaction and recrystallized with ethanol which gave the dendrimerized product 3 . The product was analyzed by using IR, ${ }^{1} \mathrm{H}$ NMR, ${ }^{13} \mathrm{C}$ NMR (Figures 1-3).

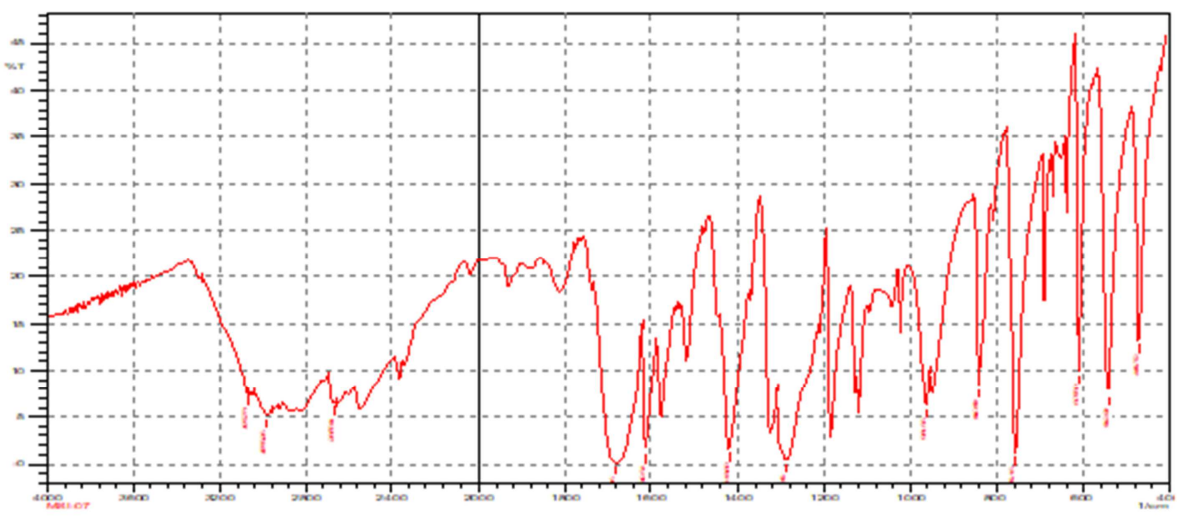

Figure 1. IR of 2,4,6-tris(di-acetamido)-1,3,5-triazine 3.

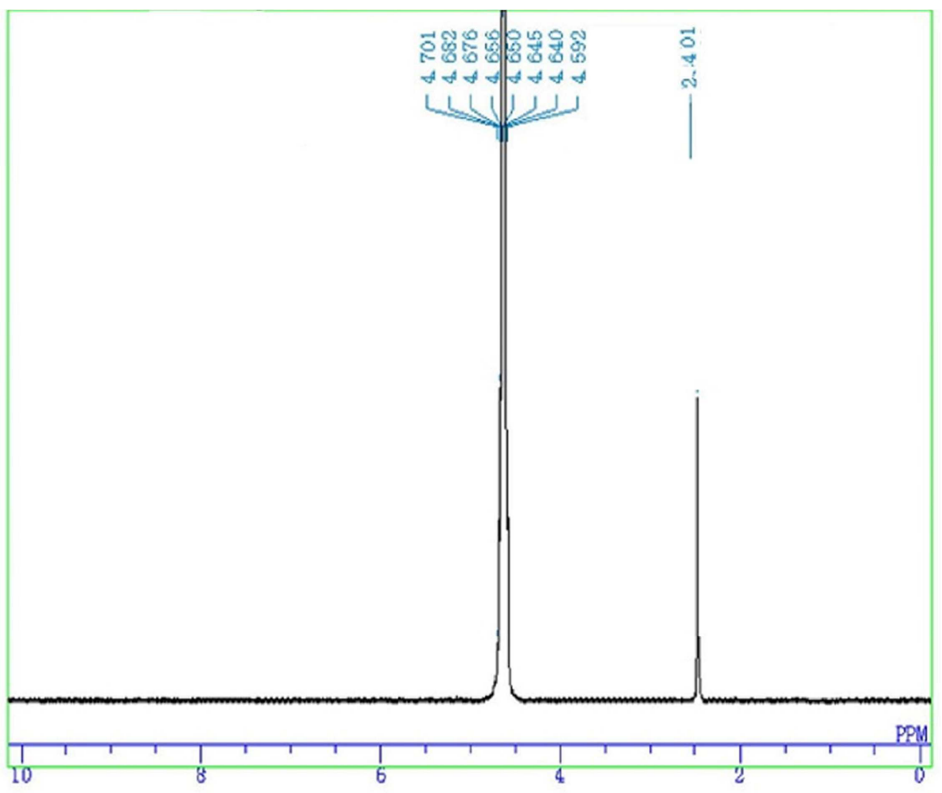

Figure 2. ${ }^{1} H$ NMR of 2,4,6-tris(di-acetamido)-1,3,5-triazine 3. 


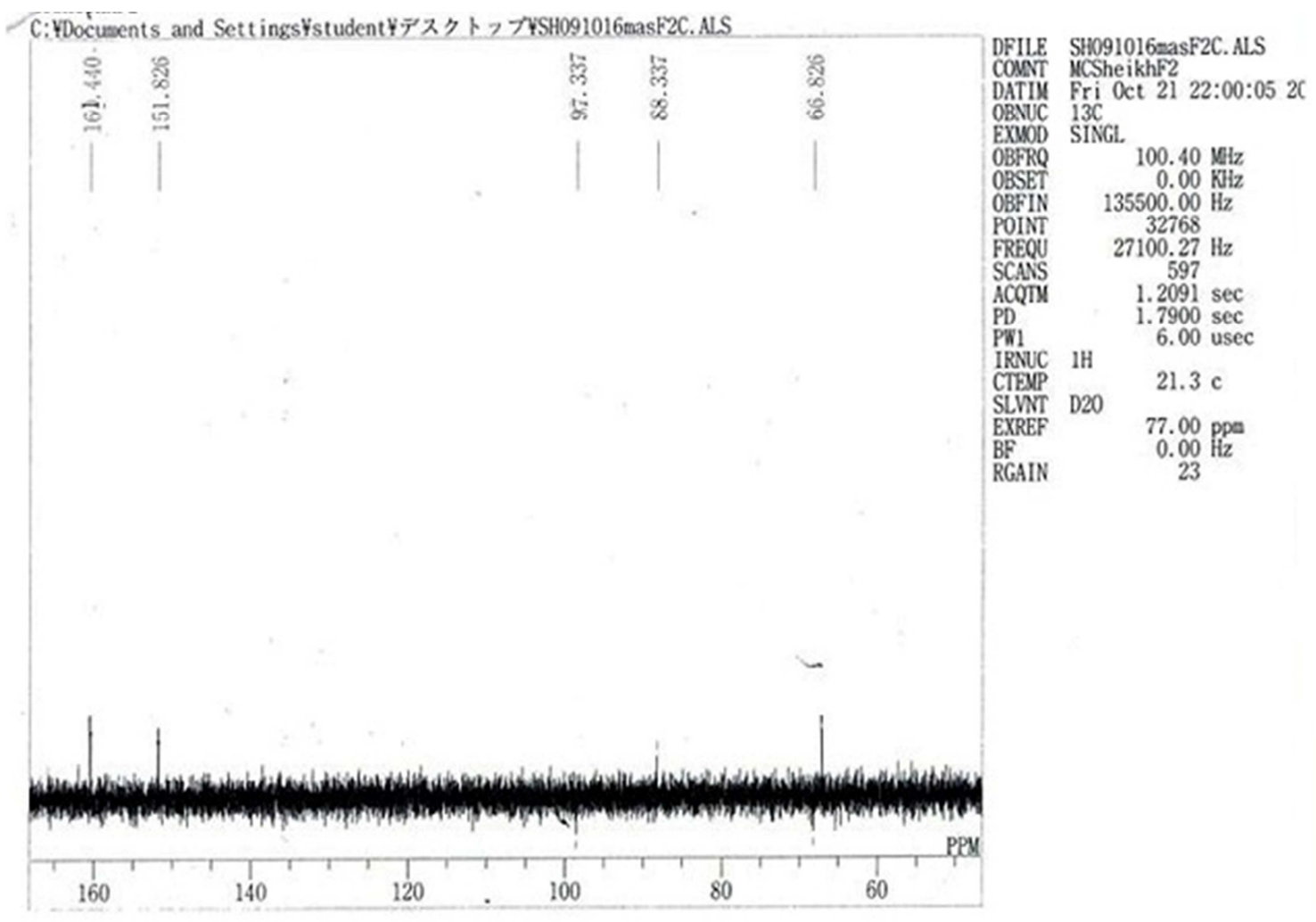

Figure 3. ${ }^{13}$ C NMR of 2,4,6-tris(di-acetamido)-1,3,5-triazine 3.<smiles>[R]C(=O)N(C([R])=O)c1nc(N(C([R])=O)C([R])=O)nc(N(C([R])=O)C([R])=O)n1</smiles>

Figure 4. Synthesis of 2,4,6-tris(di-acetamido)-1,3,5-triazine 3.

The presence of essential peaks in the IR spectrum revealed compound 3's structure. The peak at the 3035, 2965, and $1530 \mathrm{~cm}^{-1}$ stretching bands suggested the existence of aromatic $\mathrm{C}-\mathrm{H}$, aliphatic $\mathrm{C}-\mathrm{H}$ and aromatic $\mathrm{C}=\mathrm{C}$ respectively, while the $\mathrm{C}=\mathrm{O}$ group was ensured by the peak at $1620-1780$ $\mathrm{cm}^{-1}$. Indeed it is remarkable that there is no band for $\mathrm{N}-\mathrm{H}$ close to $3400-3200 \mathrm{~cm}^{-1}$ which has been shown to have changed $1^{\circ}$ amino group of triazine into $3^{\circ}$ amido group. The preferred dendrimer 3 was also afforded in ${ }^{\mathrm{I}} \mathrm{H}$ NMR and ${ }^{13} \mathrm{C}$ NMR spectra. There is no $\mathrm{NH}$ or $\mathrm{NH}_{2}$ group in the ${ }^{1} \mathrm{H}$ NMR peak, which further defined the structure of 3 . The structure of compound 3 was also confirmed by the elemental analysis.

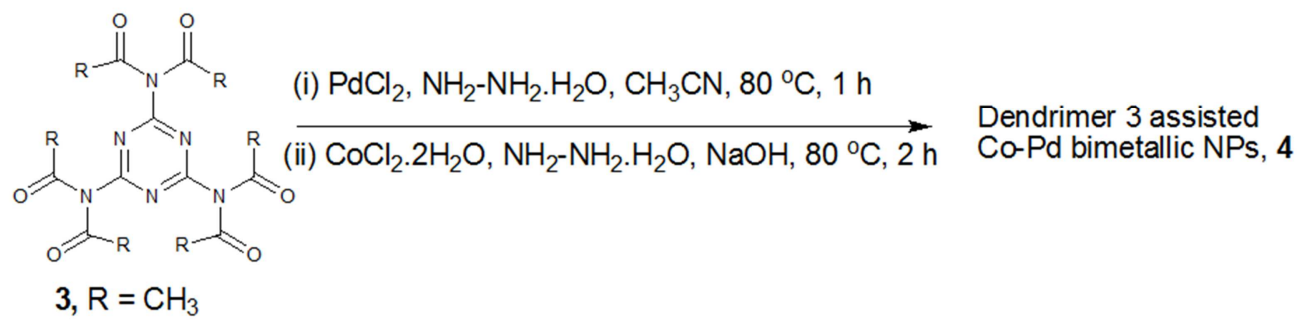

Figure 5. Synthesis of 2,4,6-tris(di-acetamido)-1,3,5-triazine assisted bimetallic Co-Pd NPs 4 .

A sequential loading approach (Figure 5) was used to produce the triazine-based dendrimer 3 assisted bimetallic Co-Pd NPs 4 and thus characterized by various physicochemical methods such as EDX, XRD, TGA, and DSC. The existence of cobalt was $55.75 \%$ and palladium $9.40 \%$ of the mass of the synthesized NPs 4 (Figure 6) was found by EDX analysis. 


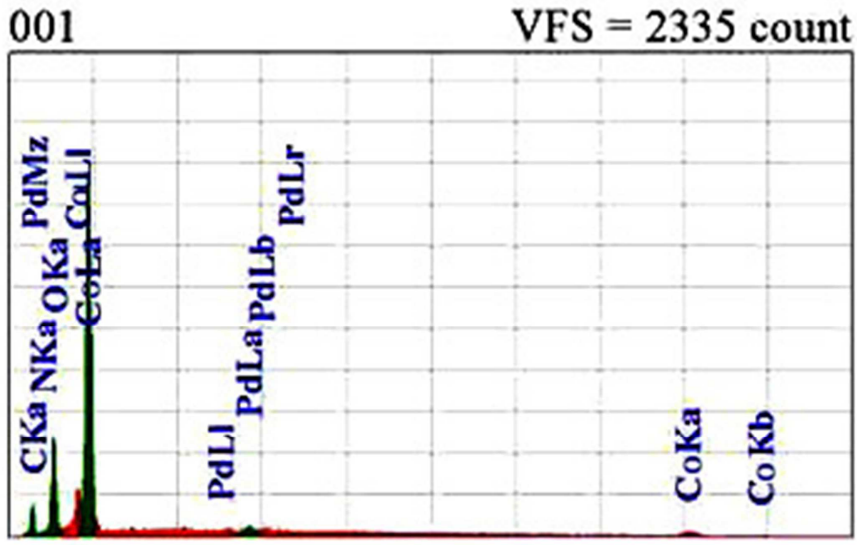

Acquisition Parameter Instrument : $7600 \mathrm{P}$ Ace. Voleage : $10.0 \mathrm{kV}$ Probe Current: $1.00000 \mathrm{nA}$ PHA mode : T3 Real Time: $30.28 \mathrm{sec}$ Live Tine : $30.00 \mathrm{sec}$ Dead Time : 0

Counting Rate: 1044 cps

Energy Range : $0-20 \mathrm{kel}$

\begin{tabular}{|c|c|c|c|c|c|c|c|c|}
\hline $\begin{array}{l}\text { Element } \\
\text { C K* } \\
\text { N K* } \\
0 \mathrm{~K} \\
\text { Co L } \\
\text { Pd L L } \\
\text { Total }\end{array}$ & $\begin{array}{l}\text { (keV) } \\
0.277 \\
0.392 \\
0.525 \\
0.930 \\
2.838\end{array}$ & $\begin{array}{r}\text { Masst } \\
14.02 \\
1.61 \\
19.22 \\
55.75 \\
9.40 \\
100.00\end{array}$ & $\begin{array}{l}\text { Sigma } \\
0.25 \\
0.15 \\
0.34 \\
0.63 \\
0.30\end{array}$ & $\begin{array}{r}\text { Atomt } \\
33.48 \\
3.30 \\
34.44 \\
22.87 \\
5.91 \\
100.00\end{array}$ & Compound & Mass \& & Cation & $\begin{array}{r}K \\
5.6763 \\
1.7853 \\
21.5728 \\
67.8324 \\
3.1331\end{array}$ \\
\hline
\end{tabular}

Figure 6. EDX analysis of 2,4,6-tris(di-acetamido)-1,3,5-triazine assisted bimetallic Co-Pd NPs 4.

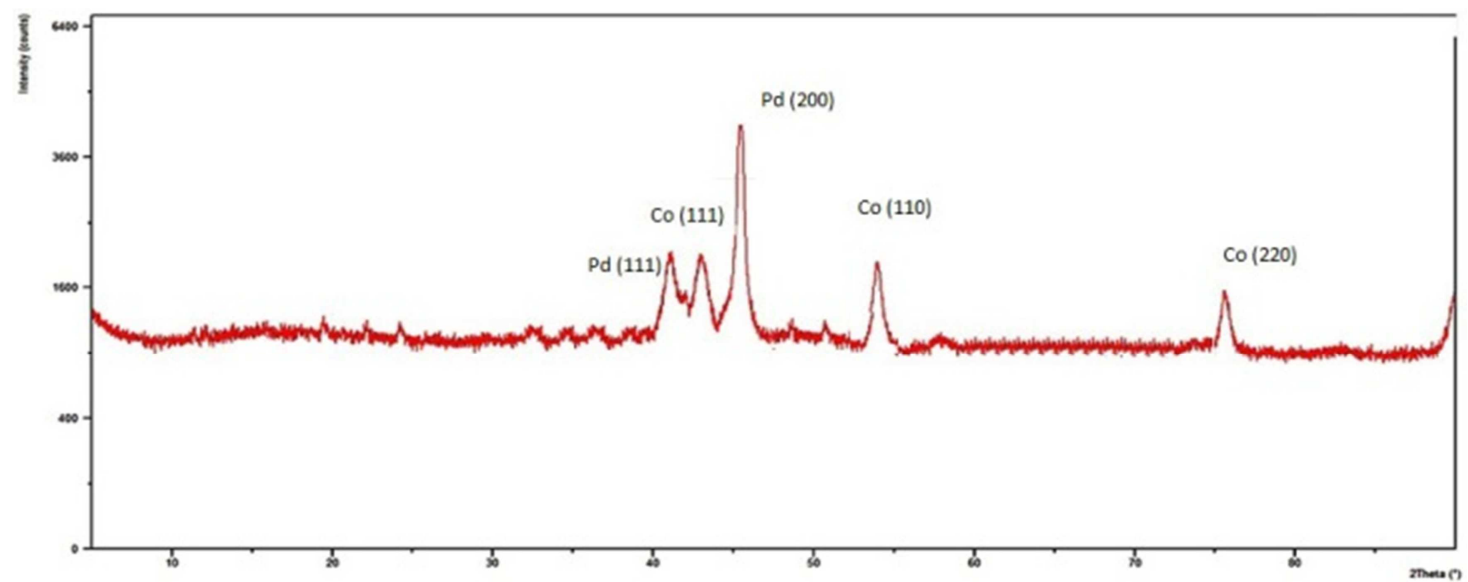

Figure 7. XRD graph of 2,4,6-tris(di-acetamido)-1,3,5-triazine assisted bimetallic Co-Pd NPS 4.

The XRD graph (Figure 7) also outlined the triazine-based Co-Pd bimetallic NPs 4 particle shape and size. Three prominent peaks of FCC Cobalt nanoparticles were related (111), (110) and (220) at $43.10^{\circ}, 54.50^{\circ}$ and $75.50^{\circ}$ respectively associated with the Co FCC JCPDS \#15-0806 standard JCPDS powder diffraction card [15] whereas the two fundamental peaks at 2 theta $^{(0)}$ values of $41.10^{\circ}$ and $45.50^{\circ}$ correlate to (111) and (200) Palladium planes respectively those represented the formation of FCC Palladium nanoparticles and, particularly in comparison to the well-known JCPDS powder diffraction card, Palladium report no (46-1043) [16]. As a consequence, these results were found in the FCC framework of triazine-based dendrimer assisted Co-Pd bimetallic NP. The dendrimer assisted Co-Pd bimetallic NPs 4 XRD pattern have been shown the average $17.50 \mathrm{~nm}$ particle length calculated through the Debye-Scherrer formula whereas TEM analysis showed the average particle size of the NPs was $14.50 \mathrm{~nm}$ (Figure 9)

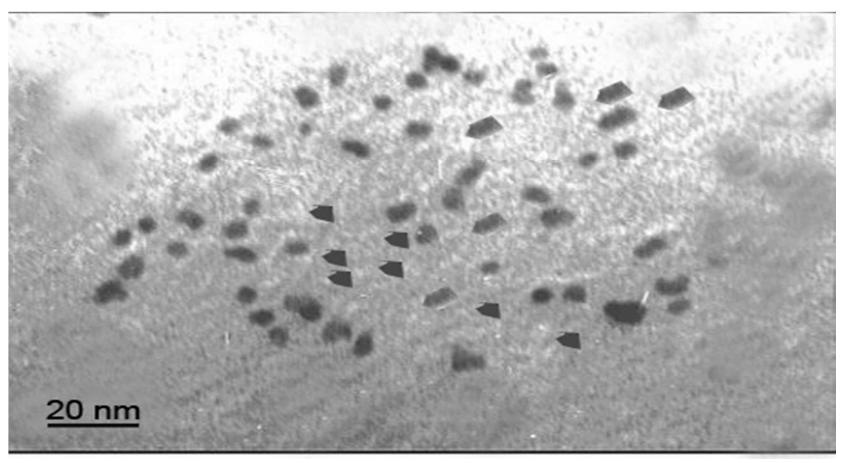

Figure 8. TEM analysis of Co-Pd bimetallic NPS 4. 


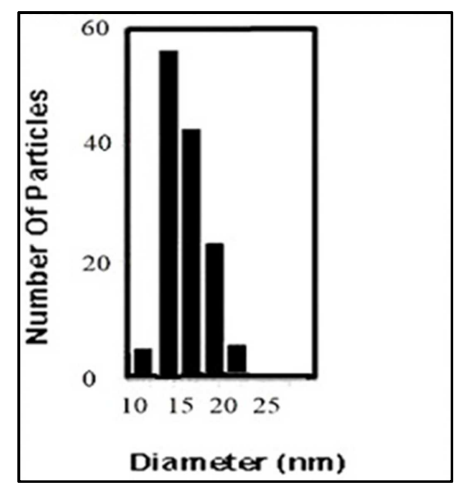

Figure 9. Particles distribution of Co-Pd bimetallic NPS 4.
The main characteristic of catalysts is thermal constancy to sustain their activity in lengthy reaction times. It has been recorded from the TG and DSC curves of Co-Pd bimetallic NPs 4 (Figure 10). Nanoparticle substrate changes with its structure and composition and is measured according to the weight loss of these elements through the thermo-gravimetric diagram. From the TG curve, it was recorded that the overall weight loss of compound 4 from $320^{\circ} \mathrm{C}$ to $347^{\circ} \mathrm{C}$ and the DSC values are just about comparable to the TG values. The melting point was indicated at approximately $320^{\circ} \mathrm{C}$ from the DSC endothermic curve. The final step of decay revealed about $15.10 \%$ ash residue of the catalyst 4 .

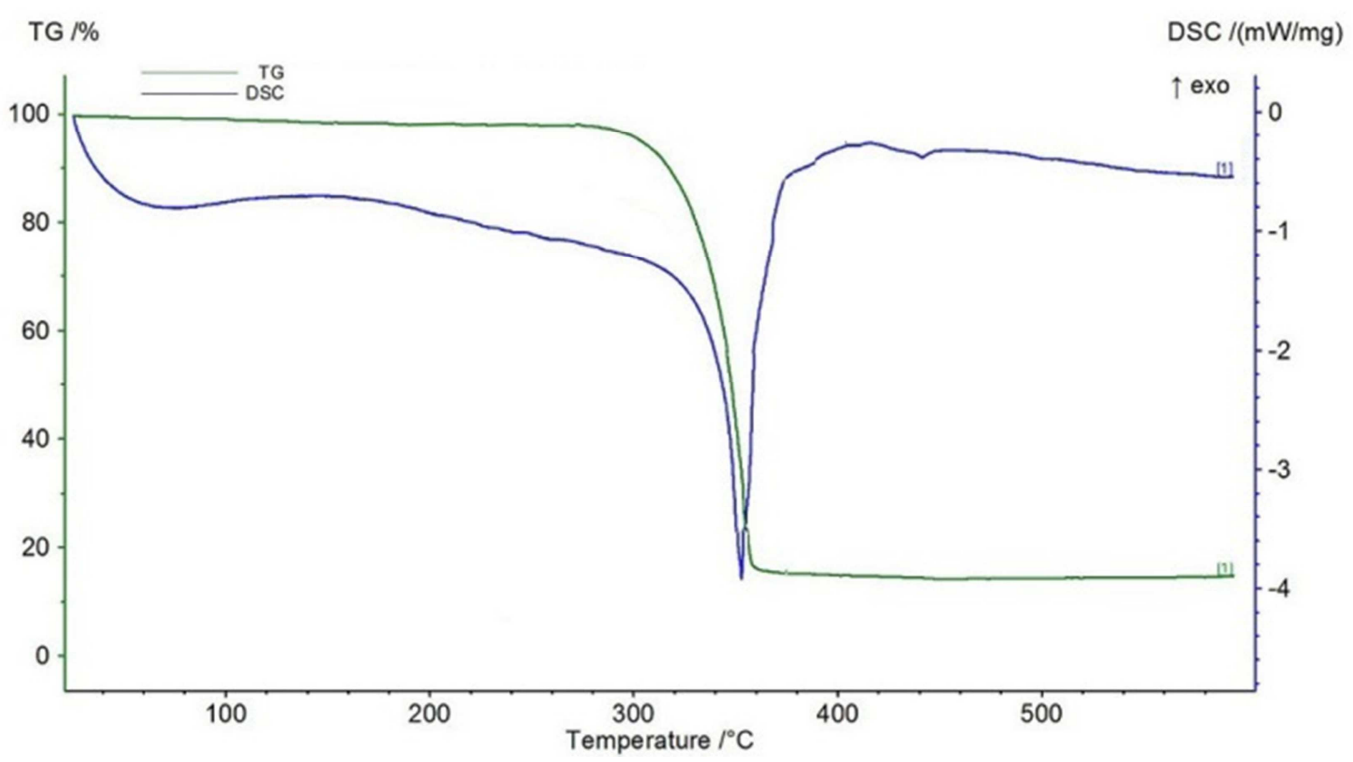

Figure 10. TG\& DSC curves of 2,4,6-tris(di-acetamido)-1,3,5-triazine assisted bimetallic Co-Pd NPs 4.

\subsection{Catalytic Performance of the Co-Pd Bimetallic NPs 4}

In order to investigate the effectiveness of the Co-Pd bimetallic NPs 4 in Suzuki-Miyaura cross-coupling reactions, we first examined 4-iodotoluene coupling with phenylboronic acid (Figure-3) to modify reaction conditions. Different bases have resulted in different activity in the current Suzuki reaction protocol (Table 1, entries 1-6), but the most effective basis in this condition is $\mathrm{Na}_{2} \mathrm{CO}_{3}$, which provides up to $90 \%$ isolated yield in $1 \mathrm{~h}$ ( Table 1 , entry 6 ). In comparison with other bases, lower yields were produced (Table 1, entries 1-3). From the literature, it is well renowned that water and alcohol play a very important role in SuzukiMiyaura reactions as a solvent [16-17]. At $\mathrm{MeOH} / \mathrm{H}_{2} \mathrm{O}$ (1:4) solvent mixture, Base $\left(\mathrm{Na}_{2} \mathrm{CO}_{3}\right)$, Catalyst $4(1.0 \mathrm{~mol} \%)$ at $70^{\circ} \mathrm{C}$ temperature about $1.0 \mathrm{~h}$, we first found $80 \%$ yield (Table 1, entry-5). When a single type of solvents such as $\mathrm{H}_{2} \mathrm{O}$, EtOH, $\mathrm{MeOH}$ or DMF was used, the product coupling was lower than the product obtained via the EtOH / $\mathrm{H}_{2} \mathrm{O}$ (1:4) solvent system (Table 1, Entry 6). Curiously, while in the presence of bases $\mathrm{KOH}, \mathrm{NaOH}, \mathrm{K}_{2} \mathrm{CO}_{3}$, solvents EtOH, $\mathrm{MeOH}, \mathrm{H}_{2} \mathrm{O}$, DMF, the temperature of up to $70^{\circ} \mathrm{C}$ and catalytic quantity of catalyst 4 was increased to $1.0 \mathrm{~mol} \%$, the yield percentage was also increased above $70 \%$ of yield at $1.0 \mathrm{~h}$ (Table-1, entries 1-4). The optimized condition and excellent yield of $90 \%$ were found in the solvent mixture EtOH / $\mathrm{H}_{2} \mathrm{O}(1: 4)$, base $\left(\mathrm{Na}_{2} \mathrm{CO}_{3}\right)$, catalyst, 3 (1.0 mol \%) of Figure 11 (Table 1, Entry 6) at $70^{\circ} \mathrm{C}$ about $1.0 \mathrm{~h}$.

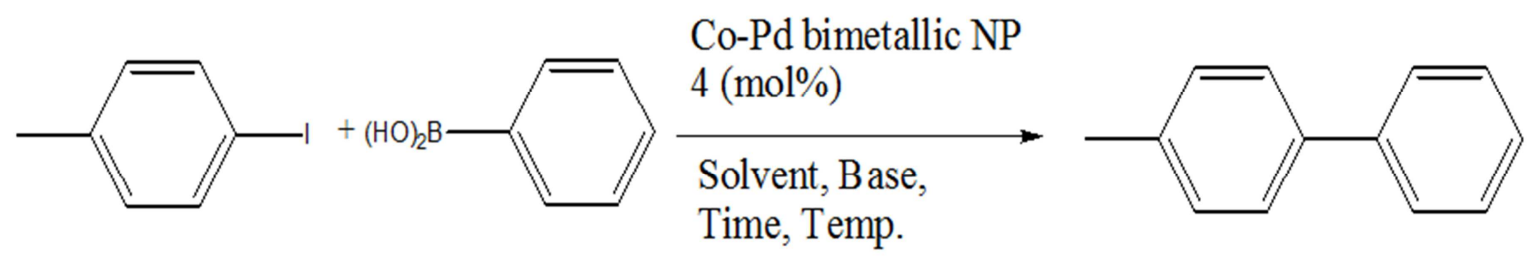

Figure 11. Optimization for the Suzuki-Miyaura reaction between 4-iodotoluene and phenyl boronic acid catalyzed by Co-Pd bimetallic NP 4. 
Table 1. Optimization for the Suzuki-Miyaura reaction between 4-iodotoluene and phenyl boronic acid catalyzed by Co-Pd bimetallic NP 4.

\begin{tabular}{|c|c|c|c|c|c|c|}
\hline Entry & Time & Temp. & Solvent & Base & Catalyst $4(\mathrm{~mol} \%)$ & Yield\% \\
\hline 1 & 1.5 & RT & $\mathrm{E}_{\mathrm{t}} \mathrm{OH}$ & $\mathrm{K}_{2} \mathrm{CO}_{3}$ & 0.5 & 25 \\
\hline 2 & 1.0 & 60 & $\mathrm{MeOH}$ & $\mathrm{KOH}$ & 0.5 & 50 \\
\hline 3 & 1.0 & 70 & $\mathrm{H}_{2} \mathrm{O}$ & $\mathrm{NaOH}$ & 1.0 & 50 \\
\hline 5 & 1.0 & 70 & $\mathrm{MeOH} / \mathrm{H}_{2} \mathrm{O}(1: 4)$ & $\mathrm{Na}_{2} \mathrm{CO}_{3}$ & 1.0 & 80 \\
\hline 6 & 1.0 & 70 & $\mathrm{EtOH} / \mathrm{H}_{2} \mathrm{O}(1: 4)$ & $\mathrm{Na}_{2} \mathrm{CO}_{3}$ & 1.0 & 90 \\
\hline
\end{tabular}

Reaction conditions: 4-iodotoluene $(1 \mathrm{mmol})$, phenylboronic acid $(1.2 \mathrm{mmol})$, base $(1.2 \mathrm{mmol})$, Solvent $(5 \mathrm{~mL})$, b) Yield \% of products is based on 4 iodotoluene.

However, in the presence of our catalytic system, different aryl iodides or bromides have been used as substrates under the above-optimized condition and all afford the corresponding products effectively. (Table 2, entries 1-5).

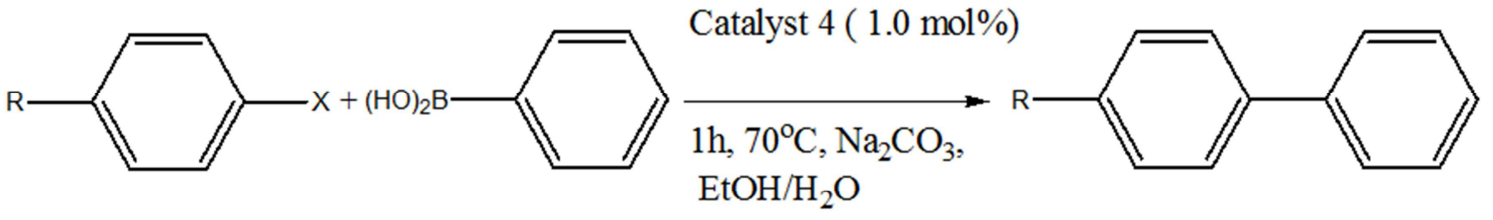

5-9

Figure 12. Differents Suzuki-Miyaura reaction under optimized conditions catalyzed by Co-Pd bimetallic NPS 4.

Table 2. Differents Suzuki-Miyaura reaction products under optimized conditions catalyzed by Co-Pd bimetallic NPs 4.

\begin{tabular}{|c|c|c|c|c|}
\hline Entry & $\mathbf{R}$ & $\mathbf{X}$ & Yield\% for cat. 4 & Products \\
\hline \multirow{2}{*}{1} & $\mathrm{H}$ & I & \multirow{2}{*}{92} & \\
\hline & 5 & 5 & & \\
\hline \multirow{3}{*}{2} & $\mathrm{H}$ & $\mathrm{Br}$ & \multirow{3}{*}{90} & \\
\hline & & & & \\
\hline & 6 & 6 & & 11 \\
\hline \multirow{2}{*}{3} & $\mathrm{CH}_{3}$ & I & \multirow{2}{*}{90} & \\
\hline & 7 & 7 & & 12 \\
\hline \multirow{2}{*}{4} & $\mathrm{CH}_{3}$ & $\mathrm{Br}$ & \multirow{2}{*}{87} & \\
\hline & 8 & 8 & & 12 \\
\hline \multirow[t]{2}{*}{5} & $\mathrm{OCH}_{3}$ & I & \multirow[t]{2}{*}{95} & \\
\hline & 9 & 9 & & \\
\hline
\end{tabular}

Yield \% of products is based on arylhalide.

All products produced through Suzuki-Miyaura coupling reactions are well known compounds and compared our acquired records with that available in the literature [14].

We also studied the recyclability of the Co-Pd bimetallic NPs 4 towards the coupling reaction between 4-iodotoluene with phenylboronic acid. During each catalytic run, black
Co-Pd bimetallic NPs 4 was recovered from the reaction mixture by filtration, washed with water and acetone, and then dried under vacuum. The recovered catalyst 4 was used for consecutives run and showed minimum decrease the yield $\%$ of products shown the following table.

Table 3. Recyclability of Co-Pd bimetallic NPs 4 for the reaction 4-iodotoluene coupling with phenylboronic acid.

\begin{tabular}{llll}
\hline Entry & Run & Time (hour) & Yield \\
\hline 1 & Fresh & 1 & 90 \\
2 & 1 & 1 & 88 \\
3 & 2 & 1 & 87 \\
4 & 3 & 1 & 84 \\
5 & 4 & 1 & 80 \\
\hline
\end{tabular}




\section{Conclusion}

In summary, we have satisfactorily developed a convenient access catalyst for various $\mathrm{C}-\mathrm{C}$ cross-coupling reactions to highly active triazine-based dendrimer supported heterogeneous Co-Pd bimetallic nanoparticles. All evaluative results (EDX, XRD, TG \& DSC) confirmed the shape and size of the nanoparticle and heterogeneous catalytic activity was also found to be phosphine-ligand-free in the carboncarbon cross-coupling reaction such as Suzuki-Miyaura coupling reactions. In addition, the introduction of high $\mathrm{Co}$ in the Co-Pd bimetallic nanoparticle catalyst not only decreased the catalyst's cost but also created it highly reusable for up to 5 catalytic cycles without any substantial loss in catalytic activity. The high recyclability catalyst $\mathrm{Co}-\mathrm{Pd}$ bimetallic nanoparticles can also be used through the formation of C-C bonds to synthesize multiple biologically active compounds. Our ongoing effort through this work will attract the scientific community's attention to developing a class of highly active bimetallic nanoparticles catalysts, so that the use of Pd for coupling reactions can be significantly reduced by introducing a second inexpensive metal (non-noble metals), while at the same time achieving stability and preserving the catalyst's heterogeneous nature.

\section{Acknowledgements}

We would like to thank the Ministry of Science and Technology, Dhaka, Bangladesh (2018-2019 National Science and Technology Fellowship Program, Ph.D. Fellowship, No- 39.00.0000.012.002.03. 8.25, Code No1260101-120005100-3821117) for providing financial support for our research work.

\section{Conflicts of Interest}

The authors declare that they have no competing interests.

\section{References}

[1] A. Archut and F. Vo"gtle, Handbook of Nanostructured Materials and Nanotechnology, H. S. Nalwa (ed.), Academic Press, San Diego, 5, 333, (2000).

[2] M. Fischer and F. Vögtle, Dendrimers: from design to application-a progress report, Angewandte Chemie International Edition, 38(7), 884-905, (1999).

[3] R. M. Crooks, M. Zhao, L. Sun, V. Chechik and L. K. Yeung, Dendrimer-encapsulated metal nanoparticles: synthesis, characterization, and applications to catalysis, Accounts of chemical research, 34(3), 181-190, (2001).

[4] R. W. Scott, H. Ye, R. R. Henriquez and R. M. Crooks, Synthesis, characterization, and stability of dendrimerencapsulated palladium nanoparticles. Chemistry of Materials, 15(20), 3873-3878, (2003).
[5] M. Pittelkow, K. Moth-Poulsen, U. Boas and J. B. Christensen, Poly (amidoamine)-dendrimer-stabilized Pd (0) nanoparticles as a catalyst for the Suzuki reaction. Langmuir, 19(18), 7682-7684, (2003).

[6] F. Zeng and S. C. Zimmerman, Dendrimers in supramolecular chemistry: from molecular recognition to self-assembly. Chemical reviews, 97(5), 1681-1712, (1997).

[7] O. A. Matthews, A. N. Shipway and J. F. Stoddart, Dendrimers-branching out from curiosities into new technologies. Progress in polymer science, 23(1), 1-56, (1998).

[8] R. Andres, E. de Jesus and J. C. Flores, Catalysts based on palladium dendrimers, New Journal of Chemistry, 31(7), 1161-1191, (2007).

[9] R. M. Crooks, M. Zhao, L. Sun, V. Chechik and L. K. Yeung, Dendrimer-encapsulated metal nanoparticles: synthesis, characterization, and applications to catalysis, Accounts of chemical research, 34(3), 181-190, (2001).

[10] W. Hui, L. R. garry, Bimetallic Dendrimer-Encapsulated Nanoparticle Catalyst. Polymer reviews, 56(3), 486-511, (2016).

[11] J. K. Nørskov, F. Abild-Pedersen, F. Studt, and T. Bligaard. Density functional theory in surface chemistry and catalysis. Proceedings of the National Academy of Sciences, 108(3), 937-943, (2011).

[12] W. M. Haynes, and D. R. Lide, 2012. CRC handbook of physics and chemistry.

[13] Y. S. Feng, X. Y. Lin, J. Hao and H. J. Xu, Pd-Co bimetallic nanoparticles supported on graphene as a highly active catalyst for Suzuki-Miyaura and Sonogashira cross-coupling reactions. Tetrahedron, 70(34), 5249-5253, (2014).

[14] Z. Zhang and Z. Wang, Diatomite-supported Pd nanoparticles: an efficient catalyst for Heck and Suzuki reactions. The Journal of organic chemistry, 71(19), 7485-7487, (2006).

[15] A. Kashif, T. Iqbal, U. S. Solat and M. Muhammad. Green synthesis of cobalt nanoparticles by using methanol extract of plant leaf as reducing agent. Pure and Applied Biology. Pure and Applied Biology 5(3), 453-457, (2016).

[16] D. Sengupta J. Saha, G. De, B. Basu, Pd/Cu bimetallic nanoparticles embedded in macroporous ion-exchange resins: an excellent heterogeneous catalyst for the Sonogashira reaction. J Mater Chem A 2(11), 3986-92, (2014).

[17] S. Yaşar, Ç. Şahin, M. Arslan, and İ. Özdemir, Synthesis, characterization and the Suzuki-Miyaura coupling reactions of N-heterocyclic carbene-Pd (II)-pyridine (PEPPSI) complexes. Journal of Organometallic Chemistry, 776, 107112, (2015).

[18] C. A. Fleckenstein, and H. Plenio, Highly efficient SuzukiMiyaura coupling of heterocyclic substrates through rational reaction design. Chemistry-A European Journal, 14(14), 4267-4279, (2008). 